\title{
ESPACES WEB POUR UNE SÉMIOTIQUE VISUELLE NUMÉRIQUE
}

\section{WEB SPACES FOR A DIGITAL VISUAL SEMIOTICS}

EVERARDO REYES*

RÉSUMÉ: Dans cette contribution nous proposons de regarder le web comme un environnement approprié à la recherche en sémiotique visuelle. Nous pensons à l'emploi de récents développements en traitement et visualisation de données sur le web. En effet, le web est devenu une plateforme puissante pour concevoir des espaces interactifs. On peut identifier ces espaces à des genres et types de sites web, mais nous croyons qu'il est possible de créer des espaces originaux inspirés directement des pratiques et méthodologies sémiotiques. Un tel espace s'avère complexe dans la mesure où il sert comme interface utilisateur pour manipuler les contenus, mais aussi, dans un deuxième temps, comme contenu lui-même. Nous évoquons la nécessité de construire une sémiotique numérique, c'est-àdire une sémiotique qui utilise les technologies numériques comme objet d'étude, comme espace d'énonciation, et comme environnement pour créer des nouvelles interfaces graphiques inspirées des méthodes sémiotiques.

* Maître de conférences à l’Université Paris 8 - Laboratoire Paragraphe.

E-mail : ereyes-garcia@univ-paris8.fr. 
MOTS-CLÉS: Interface graphique. Visualisation. Sémiotique visuelle. Données numériques.

ABSTRACT: In this paper, we consider the web as a pertinent environment for studying visual semiotics. We observe the application of recent developments in information processing and data visualization on the web. Indeed, the web has become a solid platform to interactive space design. At first glance, we can identify these spaces to website types and genres, but we believe it is also possible to create original spaces directly inspired on semiotic methodologies and practices. Moreover, such spaces have been proved complex because they serve as graphical interface to manipulate the content, but also as the content itself. At the end of the paper, we stress the importance of considering a "digital semiotics" line, for instance, a semiotic approach that employs digital technologies as object of study, enunciation space, and environment to create new graphical interfaces grounded on visual semiotics.

KEYWORDS: Graphical interface. Visualization. Visual semiotics. Digital data.

\section{Le web comme plateforme de production}

On identifie les origines du web en 1991, lorsque Tim Berners-Lee introduisit la spécification du World Wide Web (BERNERS-LEE, 1999). D'un point de vue technique, l'innovation de Berners-Lee consistait à réunir le fonctionnement de trois technologies principalement: la décomposition de messages en petits paquets électroniques pour les transmettre à des adresses fixes dans un format 
adapté. Ainsi, le protocole HTTP (de l'anglais HyperText Transfer Protocol) permet l'envoi des paquets; l'URL (Uniform Resource Locator) définit les adresses universelles des ressources; et, le langage HTML (Hypertext Markup Language) constitue le format des données.

À l'origine le web avait été conçu pour un usage scientifique et documentaire. L'idée était de permettre à des chercheurs l'échange rapide et à distance des ressources documentaires. Il est possible de constater cette vision lors d'un aperçu rapide au langage HTML.

Le langage HTML est un langage nommé de balisage.

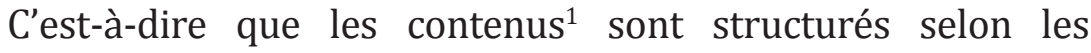
balises prédéfinies par le langage. Dans ce sens, par exemple, HTML propose de structurer les contenus textuels selon des organisations schématiques : entête, article, titre, sous-titre, paragraphe, tableau, bloc de citation, etc. Depuis la première version de HTML, apparue en 1993, la quantité et types de balises ont évoluée ${ }^{2}$. La version courante est HTML5 contient 130 balises et elle a été adoptée comme recommandation par le W3C (World Wide Web Consortium ${ }^{3}$ ) en octobre 2014 (W3C, 2014).

Par sa structure schématique, les documents web

1 Le terme «contenu» sera ici entendu dans un sens différent à celui utilisé par Hjelmslev et Greimas. En sciences de l'information et de la communication, les contenus se distinguent du médium. Ce sont les informations, les textes, les images, les ressources dans un médium quelconque.

2 La recommandation HTML5 inclut d'autres balises dédiées aux styles graphiques, contenus médias (images, vidéo, audio), et codes de programmation. Le nom et syntaxe de balises, par exemple $<\mathrm{p}></ \mathrm{p}>$ pour désigner un paragraphe est spécifié dans un langage supérieur qui s'appelle XML (eXtensible Markup Language), lui même inspiré d'un langage plus ancien nommé SGML (Standard Generalized Markup Language).

3 Le World Wide Web Consortium est l'organisation internationale qui s'occupe de définir et maintenir les standard web. Tim Berners-Lee est le directeur et fondateur. Depuis 1994, et au mois d'août 2015, regroupe 391 institutions (universités, entreprises, ONG, gouvernements, individus). http://www.w3.org/>. 
ne sont pas étrangers à une histoire de textes imprimés. Cette évolution, qui a commencé avec les codex et papyrus, a graduellement donné forme à nos supports textuels contemporains : livres, dictionnaires, encyclopédies, catalogues, brochures, magazines. Ces supports, tout comme les sites web, ne sont pas uniquement des objets, il peuvent être conçus comme des interventions temporaires dans un continuum complexe qui actualise des «conversations en réseau, de versions, de champs de débat, de références à travers une longue variété de temps et lieux» (DRUCKER, 2014, p. 175).

Avec les technologies numériques et en réseaux de nos jours il est possible d'envisager une continuité dans l'évolution des formats et des supports. De manière similaire aux pratiques des écrivains qui ont fait évoluer les supports imprimés au troisième et quatrième siècle, les nouvelles pratiques contemporaines influencent les nouveaux développements documentaires. En effet, les manières d'étudier, d'interagir, de collaborer, de gérer les informations ne sont plus les mêmes qu'à l'ère précédente du web.

Le web a une maturation de presque vingt-cinq ans. En tant que système technique hypertexte et hypermédia, le web probablement n'a pas encore réussi à intégrer toutes les fonctionnalités et visions des pionniers tels que Vannevar Bush (BUSH, 1945) ou Ted Nelson (NELSON, 1965), mais le web a certainement développé sa propre identité et son mode d'existence. Parmi ses caractéristiques, citons premièrement l'architecture client-serveur, qui permet le stockage, échange et gestion de ressources entre des ordinateurs connectés à distance. Deuxièmement, les bases de données et les langages dynamiques (comme MySQL et PHP), qui permettent d'interroger un corpus d'information et d'afficher les résultats sous format HTML. Ensuite, les navigateurs web, devenus les 
points d'accès aux informations en ligne et qui représentent, pour nous, les développements les plus stimulants et encourageants.

Si du côté serveur le web existe dans le «cloud» et dans les bases de donnés délocalisées, du côté client le web existe surtout dans les navigateurs. Le premier navigateur devenu très populaire chez les utilisateurs a été Mosaic, développé par Marc Andreessen et Eric Bina en 19934. Le succès de Mosaic a été dû surtout à sa facilité d'installation, à sa prise en main rapide et à sa capacité d'afficher des images et ressources graphiques. Dans le temps, les développeurs de Mosaic ont créé Netscape Navigator, qui à son tour est devenu la base de Mozilla Firefox. Actuellement, les navigateurs les plus populaires sont Chrome, Firefox et Safari. Cette popularité s'explique par les fonctionnalités proposées aux utilisateurs. De nos jours, la majorité des documents web exigent pour leur correcte visualisation que le navigateur intègre des technologies telles que HTML5 (au moins les balises canvas, video, audio, meta par exemple), CSS3, et JavaScript ${ }^{5}$.

Le web est devenu aussi un fournisseur de «services», au sens informatique du terme, comme on peut le constater à travers la géolocalisation et les API (Application Program

4 Le site officiel du navigateur Mosaic est: 〈http://www.ncsa.illinois.edu/enabling/mosaic〉.

5 Il est possible d'avoir un aperçu d'autres technologies en cours de développement. Les versions beta des navigateurs, comme Chrome Canary ou Firefox Beta permettent de tester des avancements en WebRTC (pour les transmissions vidéo en temps réel), Web Audio API (pour la gestion de ressources audio), WebGL (pour les graphiques 3D), WebVR (pour la réalité virtuelle et augmentée). 
Interface $)^{6}$. Dans ce cas, le web peut exister uniquement dans un mode d'existence virtuel car c'est la navigation et les actions d'un utilisateur qui l'actualise. Un même site web visité par deux personnes différentes (ou à deux moments différents de la journée) peut afficher des informations complètement distinctes car il prend en compte la géolocalisation, les sites consultés précédemment, les préférences et les paramétrages personnels obtenus des navigateurs.

Les sites web deviennent aussi un objet à feuiller. Il est possible de récupérer les informations d'un site web sans se rendre directement à son adresse URL, en utilisant un «agrégateur» de flux RSS (Really Simple Syndication) ${ }^{7}$. On peut également convertir un site en base de données sous format CSV (Comma Separated Value) ou JSON (JavaScript Object Notation) pour ensuite faire de l'analyse des textes, calcul statistiques et visualisations ${ }^{8}$. Ainsi, le web pensé comme service dessine une idée où il est dynamiquement restructuré, refaçonné, découpé en petits morceaux, requeté et interrogé, trié et ordonné.

Les manières dont l'information est traitée par les technologies en réseau sont couplées avec les supports techniques d'affichage. Dans cette contribution, nous nous concentrons sur le web existant sur les navigateurs web d'un ordinateur classique. Mais rappelons que les navigateurs existent aussi sur les téléphones portables, les tablettes, les kiosques multimédia, etc. De plus, le web constitue seulement

6 Une API est une manière dont un site web partage ses informations à des tierces personnes. C'est-à-dire que les données d'un site sont mises à la disposition à d'autres applications informatiques. De cette manière, il est possible de créer un site web qui bénéficie des données et des fonctionnalités provenant d'autres sites. Ce mode de mélanger les contenus et les fonctions est la base des nommés «mashups».

7 Pensons au populaire Feedly : http://feedly.com

8 Ceci est possible avec des outils comme artoo.js (https://medialab.github.io/artoo/) ou Kimono Labs (https://www.kimonolabs.com/). 
un élément parmi toute la variété de l'écologie internet contemporaine. L'internet s'est étalé aux consoles de jeux vidéo, à la télévision, à l'architecture et bâtiments, à l'espace publique, à l'environnement, au cosmos, et en général à tous les objets 9 . Une étude plus approfondie sur le web devrait prendre en considérations ces éléments.

\section{Les espaces web}

Nous avons vu qu'un site web est un objet complexe. Il peut être étudié comme objet, comme technologie, comme intervention temporaire, comme code de balisage et programmation. Dans cette section, nous allons considérer le web comme un espace de médiations. Expliquons nous. La face substantielle et visible d'un site web détermine un espace au sens topologique. C'est-à-dire qu'il est délimité par une surface (la fenêtre du navigateur et l'écran de l'ordinateur) où des composants graphiques donnent forme à des organisations schématiques. Dans cette optique, l'espace d'un site web est un agencement méréologique.

D'un autre côté, l'espace donné à l'utilisateur est luimême la représentation d'une série de décisions que le producteur a prise lors de la création. Un site web peut être vu comme un espace d'énonciation. Les actes de débrayage du producteur conjuguent des éléments préexistants et déterminés par les technologies web mais il les dispose et joue avec leur aspect en imaginant les actes d'embrayage de l'utilisateur. Il existe d'ailleurs une technique chez les designers web professionnels qui consiste à enregistrer les actions des

9 Pensons à l'«Internet des objets», ou IoT (Internet of Things), qui s'appuie en grande mesure sur des capteurs, senseurs, microcontrôleurs et microprocesseurs. 
visiteurs effectuées sur un site pour pouvoir proposer des mises à jour plus efficaces et adaptées à leurs «besoins» et expériences de navigation (zones où l'on clique, durée de la visite, vitesse de défilement de la page, etc.). Le producteur ouvre des possibilités techniques, actancielles, et narratives en même temps qu'il en ferme d'autres. En outre, le site web manifeste une idéologie au travers les choix hiérarchiques d'organisation de l'information, de la charte graphique, des types de médias, et du style de rédaction employé ${ }^{10}$.

Nous qualifions les sites comme espaces web pour faire analogie avec un monde virtuel. Chaque site web est comme un monde ou un lieu où nous vivons un moment. Certains mondes nous permettent de lire, de visionner une vidéo, de jouer, de manipuler un schéma, d'acheter des produits, de lancer de recherches. Ces actions ensemble constituent une expérience. C'est l'expérience, et les traces qui imprègne dans l'esprit, ce qui permet de voir le site web comme un monde. Après une session de navigation, quelle émotion ressentonsnous? Avons-nous appris quelque chose? Avions-pu faire tous ce qu'on a eu envie de faire? Avait-il eu des fonctionnalités qu'on aurait souhaité avoir à la main?

Le fait de «sauter» d'un site à un autre n'est pas uniquement une métaphore spatial, mais aussi temporelle. Physiquement, les fichiers informatiques qui constituent un site web peuvent exister dans le disque dur d'un serveur en France, tandis qu'un autre site réside au Brésil ou Japon. De plus, ces mondes ont des versions. Temporellement, on peut aller d'un site crée aux années 90 vers un autre réalisé aux

10 On peut signaler que le choix d'un langage de programmation est aussi un choix idéologique. Alors qu'une animation graphique peut être produite avec le langage ActionScript (conçu par Macromedia en 1998 et acheté par Adobe en 2005), la tendance actuelle, influencée par le mouvement open source, est plutôt de choisir JavaScript, SVG (Scalable Vector Graphics), Java, Perl, Python et HTML5 canvas. 
années $2000 s^{11}$. Enfin ces mondes peuvent avoir une durée déterminée. Il arrive qu'une fois que nous avons quitté un site, celui-ci cesse d'exister; les boutons de navigation ne permettant plus de revenir dans le temps. On peut donc imaginer que les espaces web existent dans un espacetemps plus grand, constitué de l'ensemble virtuel et possible d'espaces web ${ }^{12}$.

Cet espace serait comme un environnement où les seuls points en commun se trouvent au niveau technique, c'est-àdire les technologies derrière tous les documents web. Mais pour préciser un peu plus, on doit rappeler que la maitrise de ces technologies appartient plus généralement au domaine des sciences informatiques. Bien que plusieurs personnes provenant d'autres disciplines s'intéressent à apprendre les technologies web, le nombre reste assez restreint pour expliquer la démultiplication de sites de nos jours. Nous croyons que l'un de développements qui a aidé à assouplir les contraintes des compétences techniques sont les nommés CMS (Content Management Systems) ${ }^{13}$.

Parallèlement, les points de convergence trouvent des points de dissociation surtout au niveau de la médiation.

11 Citons au passage le projet non-lucratif Internet Archive, qui propose un module nommé Wayback Machine pour naviguer les versions anciennes d'un même site. Actuellement, le site a sauvegardé 432 millions de pages web: 〈https://archive.org/web/〉.

12 La quantité de sites web reporté par Google en 2008 était de 1 trillion (GOOGLE, 2008). À l'heure actuelle, le web maintien sa croissance exponentielle. Par ailleurs, il ne faut pas oublier qu'ils existent bien plus des sites que ceux répertoriés par Google. Le «deep web» fait référence à ceux qui ne sont pas accessibles à tout le monde mais nécessitent des mots de passe et d'autres types de sécurité de connexion.

13 Un CMS propose une interface graphique d'utilisateur dédiée à la gestion de contenus. Au lieu de gérer une base de donnée depuis une interface PHPMyAdmin par exemple, un utilisateur dispose de formulaires pour créer, modifier, effacer et publier des contenus. Dans ce sens, le sites web de réseaux sociaux comme Facebook suivent la même logique : faciliter aux utilisateurs le publication et partage d'information. Parmi les CMS les plus utilisés actuellement nous trouvons: WordPress (https://wordpress.com/website/), Joomla (http://www.joomla.org/), et SPIP (http://www.spip.net/). 
Pour aborder brièvement cette question, regardons la variété d'usages des sites web comme des genres. En effet, les composants graphiques et conventions des sites web peuvent se formuler en différents discours. Il n'est pas notre intention de citer tous les genres de sites web, toutefois listons quelques-uns : personnels, académiques, institutionnels, sites de commerce électronique, d'archivage, commerciaux, artistiques, de réseaux sociaux, d'entrepôt des données, et encore sites automatiques générés par des robots ${ }^{14}$.

Comme on peut l'imaginer, parmi les stratégies nécessaires pour qu'un site remplisse les attentes d'un certain genre, il doit simuler et intégrer ses propriétés et éléments basiques. Par exemple, un site web de commerce propose en général une liste ou galerie d'objets à acheter, des sélecteurs de quantité, un panier d'achats, un module pour entrer des informations bancaires, etc. Lors d'une session de navigation, on peut parcourir linéairement une dizaine de sites de différents genres, on peut également passer d'un genre à un autre, on peut même naviguer plusieurs sites de différent genre en même temps (grâce aux onglets de navigateurs). L'ensemble virtuel et possible d'espaces web s'avère donc hétérogène.

Un site web peut simuler un objet ou une expérience du monde physique. Un terme souvent employé pour expliquer ce phénomène est celui de la «rémédiation» (ou «rémédiatisation») proposé par Jay Bolter et Richard Grusin (BOLTER; GRUSIN, 2000). Pour ces auteurs, les nouveaux médias «rémédient» les anciens en intégrant

14 Un robot dans ce contexte sera entendu comme un script ou code de programmation qui exécute une série de tâches de manière récurrente. Un exemple de robot sont les scripts de Google basé sur l'algorithme PageRank, permettant au robot de naviguer le web à haute vitesse afin d'indexer les contenus. 
certains propriétés dans leur espace. À son tour, les «vieux médias» peuvent eux également intégrer des propriétés des nouveaux. L'analyse de Bolter et Grusin met l'accent sur les interfaces graphiques (ce qu'ils appellent la logique de l'«hypermédiation», et ce que nous avons identifié comme médium au début de ce texte) et l'expérience d'immersion médiatique (la logique de l' «immédiation» ou aussi le contenu en termes d'information-communication).

Bien que la plupart des sites reproduisent ou simulent l'existant des médias physiques, anciens ou électroniques, il est plus important de signaler que les genres web sont vivants, c'est-à-dire que ce sont nos pratiques qui peuvent les modifier et transformer. On peut modifier les genres en ajoutant ou supprimant des fonctionnalités, mais on peut aussi songer à créer des nouveaux genres. Nous reviendrons plus bas sur cette question car nous considérons que les sites web sont aussi des espaces de médiations d'idéologies, de manières de faire et d'expériences culturelles.

\section{Espaces web pour une sémiotique visuelle}

Nos pratiques et modes d'utilisation de l'information changent. Nous créons des objets techniques suivant nos nouvelles pratiques et, en même temps, ces objets nous façonnent à leur tour. Ceci n'est pas nouveau. Dans les mots de Marshall McLuhan: «We shape our tools and thereafter our tools shape us» (McLUHAN, 1964, p. xxi). Cette dialectique s'observe clairement dans le web. Par exemple, au fur et à mesure que l'on crée plus d'informations, il devient nécessaire d'inventer des moyens pour l'utiliser. Les moteurs de recherche sont, la plupart de cas, le principal moyen d'accès à l'information. Mais le résultat d'une requête nous montre surtout des paratextes, 
c'est-à-dire des méta-informations référant au contenu, et pas le contenu lui-même. Un moteur de recherche est un espace web de médiation entre les contenus et les fonctionnalités que le moteur propose. Un autre exemple: à mesure que les CMS s'instaurent comme la technologie préférée pour la création de sites, ces sites deviennent tous similaires entre eux et il est alors nécessaire de personnaliser l'apparence graphique car la structure de l'information reste identique. Dans un site fait avec WordPress, c'est WordPress l'espace de médiation entre le contenu du site et la manière d'organiser l'information en articles, rubriques, et posts.

Comme nous l'avons suggéré, le web se profile comme un environnement permettant de regrouper des médias classiques et d'en créer de nouveaux. On peut penser qu'un nouvel espace web rémédie non seulement l'aspect graphique et matériel d'autres médias, mais aussi les actions, les opérations et les modèles de médiation. Nous croyons qu'une telle démarche nécessite d'investiguer des procédés complémentaires aux actuels paradigmes de design et développement web. En premier lieu, l'architecture de l'information d'un nouvel espace web peut être différente à celle d'un CMS (que ce soit WordPress, Joomla, SPIP, ou autre) donc il est souvent plus judicieux d'utiliser les technologies web directement. En deuxième instance, les paradigmes de design UX/UI (User Experience/User Interface) mettent en avant la notion de «persona». Il s'agit de penser aux «besoins» d'un utilisateur typique. Le problème avec cette vision est qu'un chercheur en sémiotique est précisément un «chercheur en sciences humaines» et pas un «acheteur» ou un «manager» ou un «utilisateur typique» qui sait à l'avance ce qu'il va découvrir. Nous pensons qu'il n'est pas recommandé d'anticiper les «besoins» et actions dont il fera appel car ce 
sont justement l'expérimentation et la manipulation qui modifieront dynamiquement son parcours de recherche.

Sur cette ligne, nous souhaitons présenter dans cette contribution nos expériences en production d'espaces web originaux et inspirés des méthodes de la sémiotique visuelle. Notre démarche a deux objectives de départ. D'un côté, la conception et production d'interfaces graphiques adaptées ou issues des pratiques de la recherche en sémiotique visuelle. D’un autre côté, constituer un corpus de données propre pour faire des essais, des analyses et des observations des prototypes.

Notre intérêt sur les interfaces graphiques nous conduit naturellement à considérer les images comme objet d'étude. Nous dirons qu'un nouveau genre d'interface graphique est celui qui mobilise les modèles graphiques et diagrammatiques existants pour en créer des nouvelles actualisations interactives. Ces images ont été nommées «image-interface» (REYES, 2015). On verra que la production de ces images demande une attention particulière au plan de l'expression des images, qu'elles soient numériques ou non (FONTANILLE, 1995; FONTANILLE; DONDERO, 2012; DONDERO; REYES, 2015).

Pour exposer nos démarches méthodologiques, commençons par esquisser le cadre théorique des recherches en sémiotique visuelle conduites par le Groupe Mu (GROUPE MU, 1992). En partant de la distinction entre une sémiotique plastique et une sémiotique figurative (GREIMAS, 1984), les chercheurs du Groupe Mu proposent la classification de trois composants de l'expression des images, à savoir : chromèmes, formèmes, texturèmes. Ensuite, chaque catégorie contient des sous-éléments. 
Tableau 1 - Unités basiques du langage visuel

\begin{tabular}{|l|l|l|}
\hline Chromèmes & Formèmes & Texturèmes \\
\hline Tinte & Direction & Unité texturale \\
\hline Luminosité & Dimension & $\begin{array}{l}\text { Lois de répétition de } \\
\text { l'unité texturale }\end{array}$ \\
\hline Saturation & Position & \\
\hline
\end{tabular}

Source: Élaboration propre. Inspiré du Groupe Mu (GROUPE MU, 1992)

Il est important de souligner ici que le plan de l'expression regarde les aspects formels et matériels des médias. C'est-à-dire, l'accent est mis sur la «matérialité» visuelle et pas forcément sur l'interprétation ou les relations entre le plan de contenu et le plan de l'expression. En accord avec le Groupe $\mathrm{Mu}$, les éléments décrits dans le tableau 1 sont valables pour toutes les images, de l'art abstrait et conceptuel à l'art figurative et réaliste. Les images peuvent contenir des degrés variables et des combinassions de chromèmes, formèmes, et texturèmes. Devant une image, le chercheur en sémiotique plastique doit abstraire sa perception de figures et objets représentés pour analyser les différentes tonalités, la quantité de tintes, les emplacements de variations, les vecteurs de direction, dimension et position, les types d'unité de textures et les modèles ou patterns de répétition.

Les études classiques en sémiotique visuelle se sont consacrées pour l'essentiel aux médias analogiques comme la photographie, la peinture, les affiches, les BD, l'architecture, etc. Le défi qui pose l'étude sur les images sur le web commence par leur constitution numérique. À ce propos, plusieurs constats doivent être signalés. Premièrement, les images numériques peuvent être des représentations des images existantes sur le monde physique, c'est le cas où le chercheur scanne une 
photographie en papier ou quand il photographie une peinture avec un appareil numérique. Cette numérisation d'images (et des médias en général) pose le défit de la perte de qualité. En effet, les appareils numériques les plus sophistiqués ne sont pas capables de numériser l'expérience et le contexte tridimensionnel d'un média analogique. Il y a toujours perte ou modification de valeurs physiques. Les pixels et les modèles de couleurs ${ }^{15}$ dont nous disposons actuellement ne peuvent pas reproduire toute la gamme de couleurs visibles à l'œil humain dans le monde physique. Nous sommes, encore une fois, restreints par les possibilités de nos outils. Quelle solution à ce défi? Soit on accepte l'état technologique actuel, soit on fait des interventions directes sur les objets techniques (plus spécialement sur le «hardware»); mais dans tous les cas il ne faut pas oublier les limitations et les conditions du numérique.

Appart la numérisation, on peut créer des images directement avec des logiciels. Dans ce cas, les images peuvent être vues non seulement comme une suite de pixels qui s'affichent à l'écran. Elles peuvent se voir aussi comme des codes informatiques, ou comme des règles mathématiques, ou comme une notation de nœuds et des lignes qui définissent une forme. Ainsi, les images de synthèse sont un objet et un texte. Nous pouvons mieux expliquer cette dualité en utilisant le parcours génératif de l'expression élaboré par Jacques Fontanille (FONTANILLE, 2008).

15 Un modèle de couleur spécifie la manière dont les couleurs sont représentées. Le modèle le plus répandu dans «le numérique» est le modèle RVB, par les sigles Rouge, Vert et Bleu. Un autre modèle aussi important mais moins répandu est le modèle HSL par les sigles Tinte (Hue en anglais), Saturation, Luminosité. 
Schéma 1 - Parcours génératif de l'expression appliqué aux images

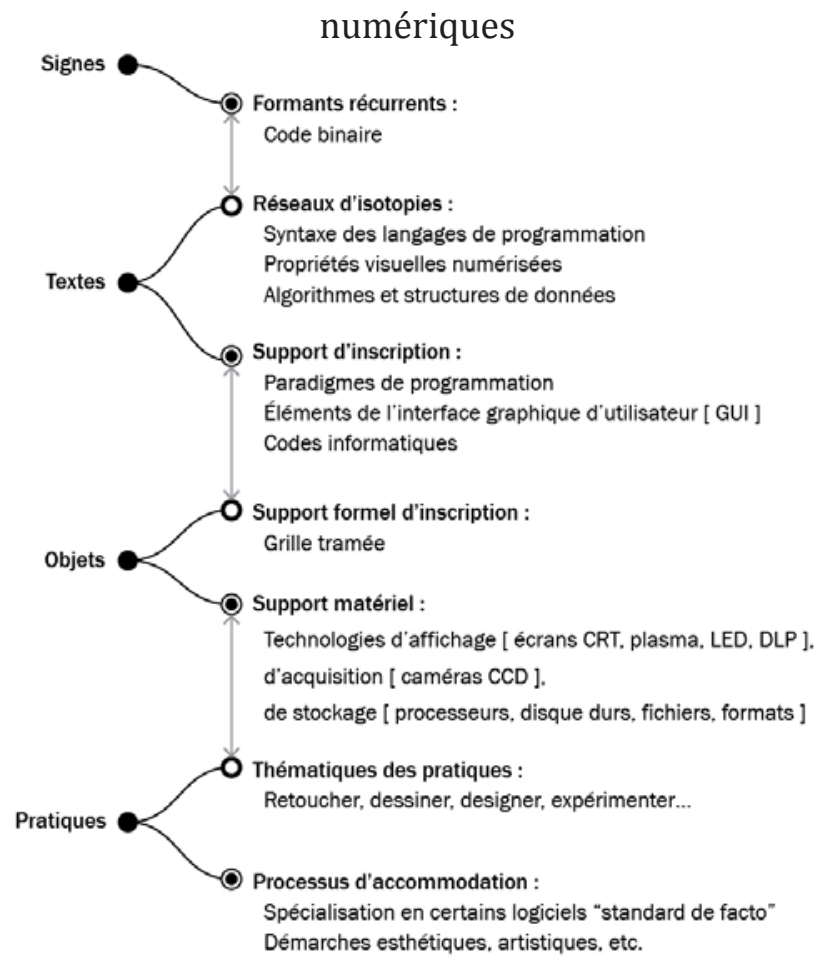

Source: Élaboration propre. Inspiré de Jacques Fontanille (FONTANILLE, 2008, p. 34)

La praticité du schéma nous permet de distinguer deux faces dans chaque niveau: une face formelle qui est plus abstraite et une face substantielle qui est plus matérielle. Les niveaux vont du plus basique (en haut du schéma) au plus complexe (en bas du schéma). À chaque niveau du parcours correspond une forme de l'expression et une substance de l'expression. Pour les images numériques, au niveau de l'objet, la forme de l'expression ce sont les supports formels d'inscription, c'est-à-dire l'omniprésente grille tramée (aussi nommée grille matricielle, en anglais raster grid) qui délimite leur structure interne. Pour sa part, la substance de 
l'expression s'associe aux supports matériels sur lesquels elles s'actualisent (écran LCD, plasma, vidéoprojecteur), se capturent (les caméras et appareil photo), et se stockent (les formats d'images comme PSD, JPG, PNG).

Si l'on observe le niveau des textes, nous identifions les supports formels des images numériques aux réseaux d'isotopies comprenant : les syntaxes de langages de programmation, les propriétés visuelles numérisées (c'està-dire les chromèmes, les formèmes et les texturèmes en version numérique), et les algorithmes et structures de données. La face substantielle serait comprise par la syntaxe des paradigmes de programmation que les langages informatiques nous permettent de mettre en œuvre, par les éléments de l'interface graphique d'utilisateur (les boutons, les champs de texte, les sliders, les menus déroulants, etc.), et par les codes informatiques comme blocs de texte écrit ${ }^{16}$.

Ces réflexions nous servent comme point de départ pour concevoir des espaces web inspirés des recherches en sémiotique visuelle. Le web en tant qu'«image-interface» est un objet composé de pixels qui s'affiche sur la fenêtre du navigateur d'un écran d'ordinateur. Ce constat nous amène à penser les dimensions dont nous disposons pour produire un espace. Si l'on regarde la résolution des écrans, on voit qu'elle a évoluée énormément, allant d'un standard de 800 x 600 pixels au début des années 2000 au plus récent 1920

16 Un code informatique est un bloc d'instructions. Pour les langages de programmations, les codes se regroupent souvent en lignes d'instructions et des fonctions. Un ensemble de fonctions donne forme à un logiciel. Un logiciel existe sous forme écrite (le code de programmation) et sous forme graphique (quand il est exécuté et compilé, en termes informatiques). La taille d'un logiciel sous forme de code informatique peut être de quelques milliers de lignes de code. 
x 1080 pixels en $2015^{17}$. En outre, les pixels existants dans cet espace peuvent se remplir selon le codage de couleur de facto de nos jours : RVB. Cette norme permet de récréer la couleur d'un pixel en combinant trois teintes : rouge, vert et bleu. Chaque tinte comprenant un octet pour représenter 255 valeurs. Ainsi, les images courantes de nos jours sont à 24-bits (8 bits par tinte) permettant d'afficher jusqu'aux 16.777.216 couleurs.

Ensuite, le niveau de texte nous rappelle qu'un espace web est déterminé par les langages de programmation, les propriétés visuelles numériques, et les algorithmes et structures de données.

Comme nous l'avons déjà indiqué, les principaux langages de programmation sur le web, du côté client, sont HTML, CSS et JavaScript. La manière dont ils se combinent pour donner forme à un site web est bien connue: le code HTML définit la structure de l'information, les sections et l'organisation schématique; le code CSS s'occupe des styles visuels, de la spécification des couleurs, positionnements, polices, etc.; le code JavaScript sert principalement à ajouter de l'interactivité aux éléments HTML et CSS. JavaScript permet de modifier l'information à la volée, d'ajouter des événements qui se déclenchent automatiquement ou manuellement. De plus, JavaScript permet la gestion des graphiques sophistiqués en manipulant l'élément HTML5 canvas, le format d'images

17 Ces données ont été prises du site W3Schools: «ttp://www.w3schools.com/browsers/ browsers_display.asp». Nous laissons de côté les différents appareils mobiles comme les tablettes et téléphones portables car nos ciblons spécifiquement des expériences avec des ordinateurs classiques. Les dispositifs mobiles méritent une étude appart en vertu de leur complexité et variété. 
vectorielles SVG, et les images 3D avec WebGL ${ }^{18}$.

Pour les propriétés visuelles numériques, nous entendons la représentation numérique des catégories du Groupe $\mathrm{Mu}$ (cf. Tableau 1). Les travaux en «cultural analytics» menés depuis 2008 par la Software Studies Initiative (SSI), sous la direction du chercheur Lev Manovich, offrent un point de départ solide (MANOVICH; DOUGLASS, 2009). En utilisant des techniques computationnelles d'extraction d'information, l'équipe de la SSI a quantifié jusqu'à 399 propriétés visuelles, regroupées en 14 catégories.

Tableau 2 - Propriétés visuelles numériques. 14 catégories regroupant 399 propriétés

\begin{tabular}{|l|c|}
\hline Catégorie & $\begin{array}{c}\text { Nombre de propriétés } \\
\text { quantifiées }\end{array}$ \\
\hline Quantification adaptative de la couleur (ACQ) & 112 \\
\hline Statistiques de base sur le canal de lumière & 7 \\
\hline Histogrammes des niveaux de gris & 40 \\
\hline Statistiques de base sur les canaux RVB & 21 \\
\hline Histogrammes des couleurs RVB & 36 \\
\hline Statistiques de base sur les canaux HSL & 21 \\
\hline Histogrammes des couleurs HSV & 36 \\
\hline Différence de bloc des probabilités inverses & 29 \\
\hline $\begin{array}{l}\text { Variation de bloc de corrélation locale } \\
\text { des coefficients }\end{array}$ & 29 \\
\hline Entropie & 1 \\
\hline $\begin{array}{l}\text { Cooccurrence de mesures des niveaux de } \\
\text { gris et mesures de matrice de texture }\end{array}$ & 16 \\
\hline
\end{tabular}

18 De fait, la gestion d'images avec JavaScript se fait le plus souvent avec une bibliothèque. L'idée est de simplifier la manipulation directe de graphiques avec l'emploi d'un métalangage. Une bibliothèque introduit sa propre syntaxe, similaire tout de même à JavaScript. Par exemple, d3.js (http://d3js.org/) est très utilisé pour les graphiques SVG 2D, tandis que three.js (http://threejs.org/) est très utile pour le WebGL et les graphiques 3D. 


\begin{tabular}{|l|c|}
\hline Energie Sobel edge & 1 \\
\hline Propriétés Gabor & 16 \\
\hline $\begin{array}{l}\text { Segmentation de couleur basée sur la } \\
\text { similarité }\end{array}$ & 34 \\
\hline
\end{tabular}

Source: Élaboration propre. Inspiré de la spécification de FeatureExtractor, un script pour MATLAB développé par la Software Studies Initiative ${ }^{19}$

Il n'est pas notre objectif ici de décrire chacune de ces catégories. Nous nous contenterons de dire que les chromèmes envisagés par le Groupe $\mathrm{Mu}$ trouvent bien leur équivalent sous format numérique (à travers les catégories sur les statistiques des canaux RVB et HSL). En ce qui concerne les formèmes, actuellement il est possible de quantifier les différentes dimensions des objets, leur complexité et leur distribution dans l'espace. La technique implique de considérer les formèmes comme des blocs de particules ou des régions d'intérêt. Par contre, il n'existe pas encore un outil d'extraction de vecteurs des formes, comme le montrent les schémas des analyses de Thurlemann (THÜRLEMANN, 2004) et Sonesson (SONESSON, 1988). Pour les texturèmes, la situation se complique car les images numériques n'on pas la troisième dimension de la texture qui est présente chez les images physiques. On peut simplement l'approcher avec une qualité de restitution supérieure ${ }^{20}$. De fait, nous dirons que la texture des images numériques est délimitée par la manière dont les pixels se rassemblent à l'écran : la grille matricielle

19 Disponible: 〈https://code.google.com/p/softwarestudies/wiki/FeatureExtractor». Consulté: 13 déc. 2015.

20 On pense en particulier au Google Art Project, qui permet de «zoomer» dans une image pour observer les grains et les détails de la texture. 〈https://www.google.com/culturalinstitute/u/0/project/art-project〉. 
qui sert de support formelle. Nous sommes devant un constat des images numériques car l'image représentée n'est pas l'objet physique, elle ne contient pas les mêmes couleurs et, en plus, son format de traitement peut réduire la qualité d'informations visuelles ${ }^{21}$.

La numérisation des propriétés visuelles va de pair avec leur traitement mathématique et informatique. Par exemple, suivant le modèle de couleur RVB, on sait que la notation de la couler rouge consiste à combiner 255 valeurs de rouge +0 valeurs de vert + 0 valeurs de bleu. Ceci peut s'écrire $(255,0,0)$ en notation RVB ou encore \#FF0000 en notation hexadécimale. En général, les producteurs peuvent choisir entre régler avec du code informatique les combinaisons de couleurs ou de faire appel à une interface graphique d'utilisateur. En termes d'interface graphique, les conventions adoptées emploient des champs de texte, des sliders, des sélecteurs spatiaux de couleur. Mais un producteur peut également concevoir ses propres manières de rendre disponibles ces valeurs. Existentt-ils des modèles ou pratiques à étudier ? C'est-à-dire, une fois que l'on a extrait des propriétés visuelles d'un corpus d'images, comment les rendre visibles et explorables aux visiteurs ? Quel modèle de visualisation adopter?

Les descriptions informatiques des données visuelles peuvent se manipuler à l'aide des algorithmes. On peut comprendre un algorithme comme une suite d'étapes, comme une recette des pas à suivre. L'idée d'utiliser un algorithme est que l'ordinateur puisse nous aider à réaliser ces actions de

21 Le format des images sur le web ce sont des formats compressés. C'est-à-dire que le poids de l'image est réduit pour une meilleure distribution sur Internet. Un format non-compressé comme TIF, ou propriétaire comme PSD n'est pas visualisé de manière native sur le web. Il est nécessaire de transformer le format de l'image en JPG ou PNG, le plus souvent. Disons en passant que le format JPG réduit l'aspect visuelle jusqu'au 30\% du champ visuel humain (CUBITT, 2014). 
manière récurrente. Un exemple basique peut être d'utiliser un algorithme pour redimensionner plusieurs images et les rendre à une taille unifiée. Un autre exemple serait d'utiliser un algorithme pour trier les couleurs d'une image selon le modèle RVB ou HSL. Récemment, avec l'arrivée du «big data», on utilise de plus en plus des algorithmes pour spatialiser les réseaux de nœuds et de liens ${ }^{22}$. On doit rappeler également qu'au moment de la production d'une interface graphique d'utilisateur, on peut penser que plusieurs algorithmes cohabitent dans un même espace. En effet, on peut utiliser un ou plusieurs algorithmes à partir de l'exécution d'un élément de l'interface graphique (par exemple l'opération «appuyer sur un bouton» peut déclencher une série de pas prédéfinis). Le résultat de cette exécution étant susceptible de traitement par un autre algorithme et ainsi de suite.

Avant de montrer quelques exemples concrets, il est souhaitable de mentionner la relation entre les algorithmes et les structures de données. En informatique, une structure de données est une manière particulière d'organiser les types de données. On ne parle pas ici d'organisation spatiale, mais des bits et bytes. Les types de données sont les unités minimales dont un langage de programmation dispose, par exemple des nombres, des chaînes de caractères, des valeurs Booleans, etc. Un exemple de structure de données ce sont les tableaux (array en anglais). Cette structure permet de stocker de manière ordonnée un ensemble de donnés pour ensuite les rendre utilisables à travers le langage de programmation. D’autres exemples de structure de données sont les listes, les arbres et les graphes. Les images « bitmap » peuvent être considérées comme des tableaux de pixels.

22 Pensons aux algorithmes de spatialisation suivants: force-based; spectral; orthogonal; tree; arc diagrams; circular layout; dominance drawing, entre autres (LIMA, 2011). 
Le principal problème que nous retrouvons par rapport à notre démarche, en termes de structures de données et d'algorithmes, est que les langages web n'ont pas été conçus à l'origine pour le traitement d'images numériques. $\mathrm{Au}$ contraire, des logiciels comme MATLAB ${ }^{23}$ ou Image ${ }^{24}$ sont eux, justement, des environnements spécialisés pour ces opérations. Notre démarche de base consiste donc à relier ces deux supports: web et logiciels d'analyse d'images. Pour nous, l'élément médiateur entre les deux est un «modèle de données». Avec ImageJ, par exemple, nous pouvons extraire les valeurs chromatiques d'une série d'images mais pour les exploiter il est nécessaire de les stocker dans un format adéquat. Imaginons que l'on met ces valeurs dans un tableau Microsoft Excel où chaque ligne représente une image traitée et les colonnes ses informations associées. Sur le web, Excel n'est pas un format adapté. Un tableau de données s'utilise mieux s'il existe sous forme de base de données, que celleci soit SQL, JSON, CSV ou TDV (Tab Delimited Value). Dans tous les cas, l'intérêt de créer son propre modèle de données est qu'il peut s'enrichir selon les attentes du chercheur: une image n'est pas uniquement décrite par les informations visuelles extraites automatiquement, elle peut contenir aussi des métadonnées pertinentes telles que titre, auteur, date, lieu, style, figures reconnaissables, etc.

Nous percevons donc plusieurs médiations. Une première médiation existe entre les images elles-mêmes et leur quantification, c'est-à-dire les données extraites de

23 MATLAB est un logiciel commercial de The Mathworks Inc. «ttp://www.mathworks. com/products/matlab/s.

24 Image est logiciel gratuit développé par Wayne Rasband au sein de la Research Services Branch du National Institute of Mental Health, Bethesda, Maryland, USA. 〈http://imagej. nih.gov/ij/>. 
propriétés visuelles. Une médiation plus profonde existe entre les supports analogiques et les images numériques (dans le cas où le producteur choisit de numériser un corpus analogique comme la photographie en papier, la peinture, etc.). Il y a une autre médiation au moment de la mise en interface: les éléments, styles et organisations graphiques pour interagir avec les données. Une autre médiation peut se prévoir lors d'une session d'expérimentation avec l'interface: supposons qu'un chercheur ait arrive à un résultat intéressant suite à des multiples manipulations de l'interface, alors doit-il pouvoir enregistrer la vue? Doit-il pouvoir la partager, l'imprimer, la commenter? Dans ces cas, le résultat expérimenté sera une médiation entre le chercheur et ses pairs lors de débats et discussions.

\section{Expériences des «images-interfaces» pour le web}

En 2009 nous avons commencé à travailler sur la «média visualisation», c'est-à-dire l'étude de corpus regroupant plusieurs images à l'aide de procédés computationnelles (MANOVICH, 2011). Le terme «média visualisation» a été proposé par Manovich en 2008 au sein de la Software Studies Initiative. $\mathrm{Au}$ niveau de la surface, l'une de principales différences entre la «data visualisation» et la «média visualisation» est que la première s'est intéressée surtout à la représentation graphique et symbolique de données 
discrètes ${ }^{25}$. La data visualisation fait recours aux modèles statistiques de traitement de données. De plus, elle prend en compte des données chiffrées mais aussi des corpus de textes (comme la lexicographie, les champs syntaxiques et sémantiques). Pour sa part, la média visualisation fait appel à l'analyse visuelle. Il s'agit d'étudier des images à travers des images. Les modèles de traitement peuvent provenir de la statistique, mais se combinent avec le traitement numérique des images. Le résultat d'une média visualisation peut avoir plusieurs formes qui commencent à se stabiliser : mosaïque d'images, balayage d'images, moyenne d'images, graphique d'images, modèle 3D d'images (MANOVICH, 2010; REYES, 2014).

Il est important de noter que les premiers travaux en média visualisation n'étaient pas destinés à une distribution sur le web. Les premières preuves utilisaient les logiciels MATLAB et ImageJ. La principale plateforme de présentation était le HIPerSpace, un arrangement synchronisé d'écrans supportant une résolution de 287 mégapixels construit au Calit2 en Californie. Ensuite, à l'issue de plusieurs collaborations internationales, la SSI s'est constitué une variété de logiciels propres : VisualSense, QTIP, FeatureExtractor, ImagePlot, ImageMeasure, ImageShapes, ImageMontage, ImageSlice, ImageToObject ${ }^{26}$. Malgré ces développements, la distribution de résultats en matière de média visualisation a été l'objet d'images statiques et de vidéos, au contraire des supports distribués et interactifs en ligne, en tout cas jusqu'à

25 Nous pensons aux schémas répandus tels que «bar chart», «pie chart», «line chart», «histogramme», «dot plot», «scatter plot». Ces modèles inspirés de la statistique sont aujourd'hui disponibles sur la plupart de logiciels de feuille de calcul ou sur des bibliothèques JavaScript comme d3.js et vega.js (http://trifacta.github.io/vega/).

26 Ces logiciels sont disponibles de manière gratuite sur le site de la Software Studies Initiative: 〈http://lab.softwarestudies.com/p/software-for-digital-humanities.html〉. 
récemment ${ }^{27}$.

Parmi plusieurs expériences et réalisations académiques et artistiques ${ }^{28}$, nous avons, en 2013, construit un corpus d'images à des fins exploratoires et pédagogiques ayant comme but sa représentation sur le web. À partir de la célèbre base de données musicale AllMusic ${ }^{29}$, nous avons pris connaissance que les éditeurs et les utilisateurs étiquettent les albums et les morceaux des artistes répertoriés. Ainsi, par exemple l'album «Highway 61 Revisited» de Bob Dylan a une notation de 5 étoiles aussi bien par les utilisateurs que pas les éditeurs du site, tandis que l'album «Six» de Soft Machine a 3 étoiles par les éditeurs et 4,5 par les utilisateurs du site. Au-delà les polémiques de critères de notation, nous avons constaté que, selon la quantité d'étoiles, certains albums ressortent comme «les plus signifiants d'un genre».

Notre méthode pour recueillir les données a été basée sur l'API d'AllMusic. En effet, afin d'assouplir la consultation du site web «à la main», en sautant d'album à un autre, nous avons lancé des requêtes automatiques directement à la base de donnée et sauvegardé les résultats. Au départ, notre

27 En février 2014, des membres de la SSI ont participé au projet Selfiecity, une média visualisation de 3.200 photographies du genre «selfie» récupérées depuis Instagram. L'un des objectifs du projet était d'explorer les corpus d'images au travers diverses méthodes hybrides de visualisation en ligne. «http://selfiecity.net/〉.

28 Ces expériences nous ont permit un entrainement en techniques et méthodes. Quelques résultats ont été montrées et discutées dans des séminaires et conférences académiques. Parmi nos choix thématiques d'images, nous citons: le film «Inception» de Christopher Nolan (REYES, 2012a) ; le design graphique de planches skateboard de la marque Powell Peralta (REYES, 2012b); l'ensemble de couvertures du magazine skateboard Thrasher (https://vimeo.com/45611894); la visualité du groupe Nirvana (REYES, 2013), entre autres.

29 AllMusic.com a été lancé en 1991 par All Media Guide. En 2007 il a été acheté par Rovi Corporation. Une API de la base de donnés avait été mis en place par Rovi en 2011. Au moment de la constitution de notre corpus, entre mars et avril 2013, AllMusic appartenait encore à Rovi. Depuis août 2013 il appartient à All Media Network, LLC, et il semble que l'API a été supprimé. < http://www.allmusic.com/〉. 
critère de sélection de données était de nous concentrer sur le genre «rock» et de constituer un catalogue des albums les plus signifiants du genre. Mais pour AllMusic le genre «rock» est connu comme «pop/rock» où se trouvent d'autres sousgenres, par exemple: «Alternative / Indie Rock», «Art-Rock / Experimental», «British Invasion», «Folk / Country Rock», «Hard Rock», «Heavy Metal».

$\mathrm{Au}$ final, pour éviter des omissions et enrichir notre corpus, nous avons décidé d'inclure d'autres genres proches du rock comme le blues, le reggae et le métal. Dans sa dernière version, notre base de données comporte 2.000 albums. Lors des premières preuves, nous nous avons travaillé surtout sur les chromèmes. Ainsi notre modèle de données a été conçu de la manière la plus simple possible. Pour chaque album nous associons les informations suivantes : titre de l'album, année de parution, nom de l'artiste, genre, image de la pochette, tinte moyenne de la pochette, saturation moyenne de la pochette, et luminosité moyenne de la pochette.

Notre base de donnée est enregistrée dans plusieurs fichiers et formats, notamment Excel et Open Refine. La raison principale de cette procédure est qu'il n'existe pas un logiciel permettant le traitement universel de données. Ce que nous avons ce sont plusieurs logiciels qui offrent différentes fonctionnalités. Pour les expériences sur le web, nous avons décidé de transformer notre tableau de données au format JSON car c'est un format natif à JavaScript et facilite sa manipulation. De plus, plusieurs bibliothèques JavaScript incluent des fonctions pour traiter le format $\mathrm{JSON}^{30}$. Pour sa part, les images ont été sauvegardées sous format JPG, qui est le format d'origine utilisé par AllMusic. Néanmoins,

30 Notamment jQuery (https://jquery.com/) et D3.js (http://d3js.org/). 
nous avons repéré quelques inconsistances: plusieurs et différents formats d'images, plusieurs et différents modèles de couleurs, et des images inexistantes mais indexées avec un URL. Ces problèmes ont été résolus manuellement et au moment du «débogage» du code.

Dans tous les cas, nos premières versions ont cherché à reproduire les techniques classiques de média visualisation, notamment le mosaïque d'images et le graphique d'images. Le résultat se montre dans les copies d'écran 1 et 2.

Copie d'écran 1 -Mosaïque d'images

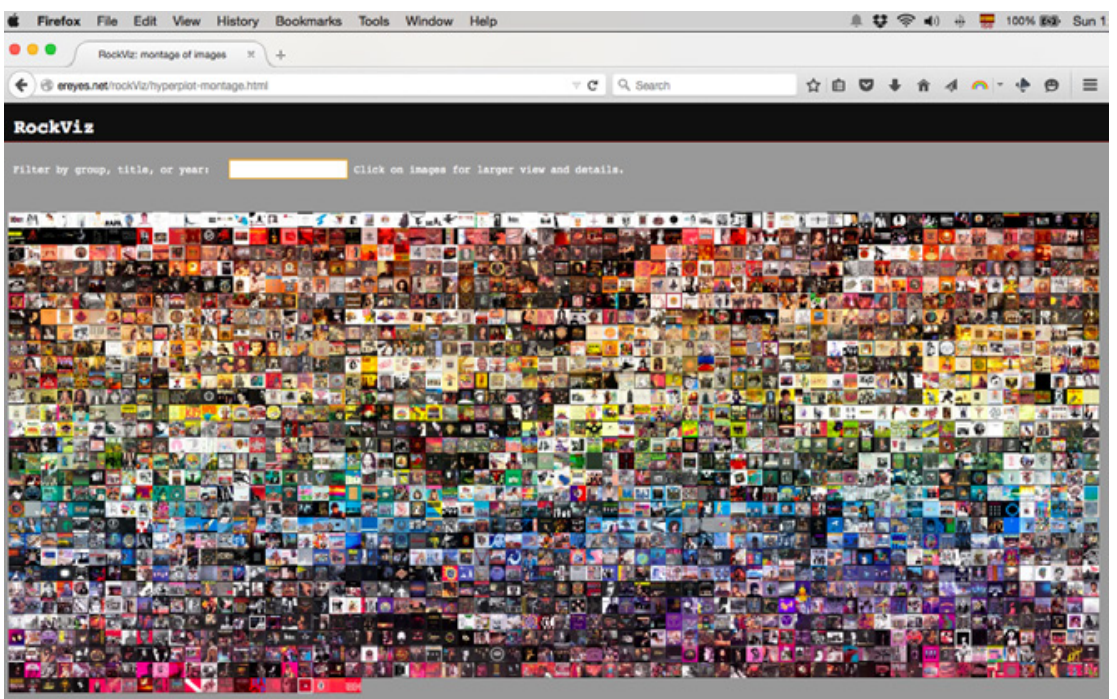

Source: Élaboration propre ${ }^{31}$.

31 Disponible: ‘http://ereyes.net/rockViz/hyperplot-montage.html». 
Copie d'écran 2 - Graphique d'images. Axe Y: tonalités couleur; Axe X: années

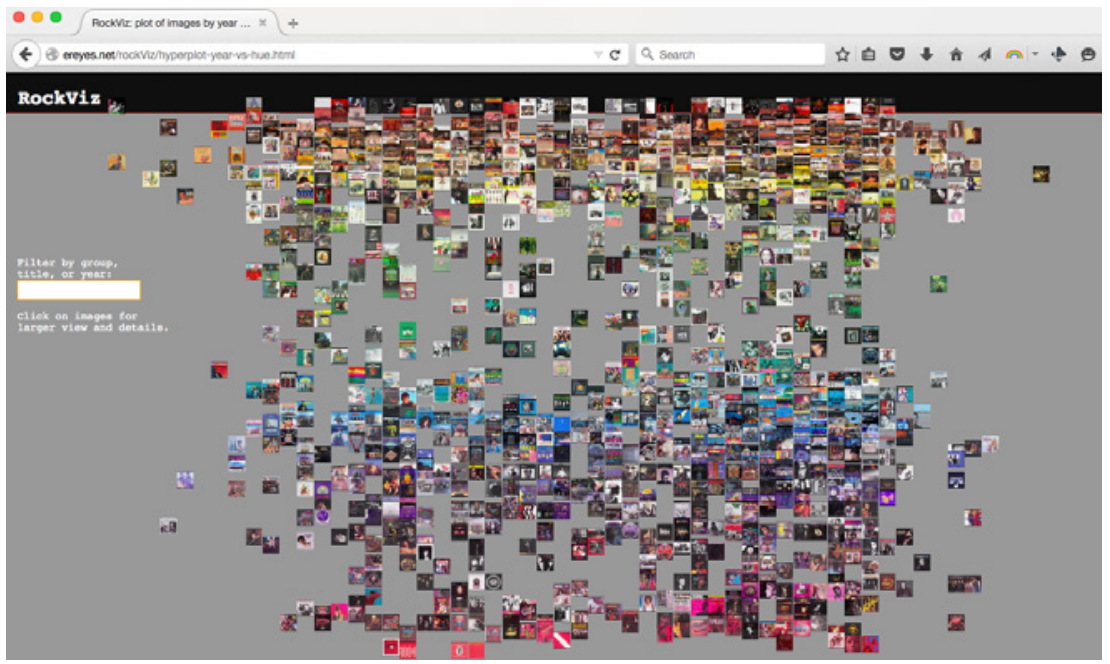

Source: Élaboration propre ${ }^{32}$.

À propos de l'emplacement des images, nous avons décidé de pré-calculer la position de chaque image et de générer automatiquement les styles CSS pour chaque fichier d'image. Pour ce faire, nous avons adapté nos propres formules mathématiques au langage GREL (qui est celui utilisé par Open Refine) afin de donner forme à un bloc de style, par exemple: «<img style=»left:70.5\%; top:4.0\%; visibility:visible;»>».

Comme on le voit, les images ont été organisées et spatialisées selon des critères définis par nous. Pour le mosaïque, les images sont placées les unes après les autres en fonction de la tinte, la luminosité et la saturation. Pour le graphique d'images, le critère sur l'axe horizontal est l'année de parution de l'album et sur l'axe vertical est la tinte. À titre

32 Disponible: «http://ereyes.net/rockViz/hyperplot-year-vs-hue.html`. 
indicatif, en langage GREL notre calcul de la position sur l'axe $X$ est «((Cells[«year»].value/1.0) - 1950) * 1500) / 1000» et pour la position sur l'axe Y est «(( (value/1.0) - 0) *3500) / $10000 »$. Dans ces formules nous avons considéré un espace visuel de la page ne dépassant pas les 1500 x 900 pixels.

En essayant de susciter des formes d'organisation originales, d'organiser différemment le corpus d'images et, éventuellement, faire surgir des patterns intéressants, nous avons modifié la formule mathématique du graphique d'images (copie d'écran 2). En effet, sur Open Refine nous avons profité du support des fonctions trigonométriques sinus et cosinus pour modifier la formule d'équations polaires : $r 2=$ $\mathrm{x} 2+\mathrm{y} 2 ; \mathrm{x}=\mathrm{r} \cos (\theta) ; \mathrm{y}=\mathrm{r} \sin (\theta)$. Un résultat peut s'apprécier dans la copie d'écran 3.

Copie d'écran 3 - Média visualisation avec variation de cosinus

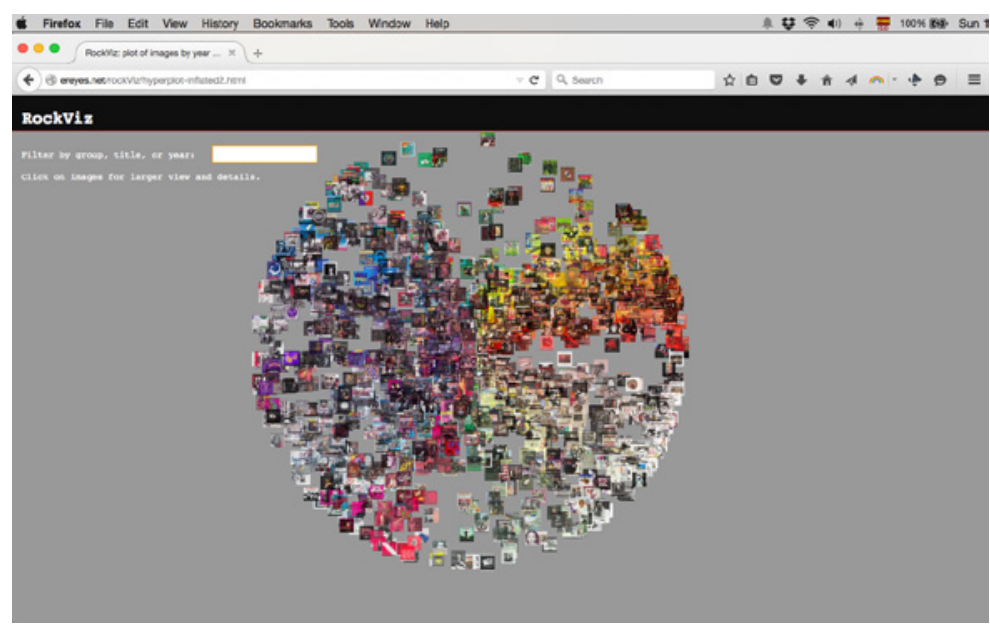

Source: Élaboration propre ${ }^{33}$.

33 Disponible: «ttp://ereyes.net/rockViz/hyperplot-radial.html». 
L'un de principaux défauts que nous avons rencontré lors de cette expérience est le temps de chargement de la page web. Techniquement, le navigateur web doit recevoir tous les fichiers JPG pour pouvoir les afficher, ce qui peut entrainer une longue durée d'attente pour les réseaux à bas débit.

Dans une expérience plus récente, nous avons continué à utiliser notre corpus d'images de pochettes de disques. Nous avons visé cette fois-ci un contexte pédagogique. Entre 2014-2015 nous avons travaillé avec des étudiants du Master 2 Design d'Interface de l'Université Paris 13 sur une réinterprétation et une «mise en interface» des données numériques.

Le cadre de départ était celui de nos expériences passées, mais afin de repenser notre démarche nous avons rétrécit le corpus d'images aux 500 morceaux les plus célèbres selon la revue Rolling Stone ${ }^{34}$. Notre modèle de données de base a évolué. Pour chaque titre musical nous avons associé : morceau, artiste, année, album, période musicale, image, luminosité moyenne, saturation moyenne, tinte moyenne, rouge moyen, vert moyen, bleu moyen, zone totale de l'image, taille moyenne de particules, formes moyennes, liens externes.

Avec un univers plus restreint et menant un travail collaboratif, nous avons constitué un corpus en réseaux et partagé. C'est-à-dire que nous avons utilisé des documents accessibles par tous les membres (étudiants et enseignant)

34 La revue Rolling Stone a publié la première version de cette liste en 2004. La liste actuelle correspond à 2010. Pour nos tests, nous avons supprimé les morceaux qui ne correspondaient pas au genre rock. De plus, l'idée proposée par l'un de nos collaborateurs, Pierre Barboza, était de rester dans les périodes 1950 - 1979. La liste officielle de morceaux peut être consulté ici: 〈http://www.rollingstone.com/music/lists/the-500-greatest-songs-of-all-time-20110407). 
depuis un navigateur web $^{35}$. Il a été intéressant de voir comment au fur et à mesure que le modèle se constituait, la base de données collective s'agrandissait tout en restant ouverte et disponible à tous les collaborateurs.

Les images-interfaces que nous avons montrées comme idées de départ n'avaient pas été raffinées intentionnellement au niveau graphique. Nous avons créée des interfaces plutôt comme des prototypes fonctionnels, voir motivationnels. Étant donné que les étudiants ont des profils et des compétences différentes, allant du design graphique aux technologies web et passant par ergonomie et management de projets, ces maquettes fonctionnelles nous aident à concentrer l'attention sur les actions disponibles, sur les résultats des manipulations, et sur la création de nouvelles versions d'interfaces.

Les copies d'écran 3 et 4 montrent deux imagesinterfaces différentes que nous avons créées comme prototypes fonctionnels.

Copie d'écran 4 - Prototype n. 01 d'image-interface

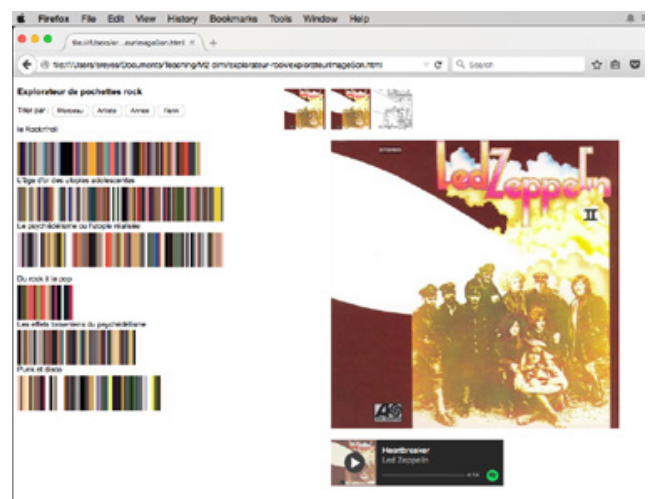

35 Nous avons choisi Google Drive pour répertorier des dossiers, créer des documents, feuilles de calculs, présentations, partager des images, liens intéressants, et d'autres ressources. «https://drive.google.com/^. 


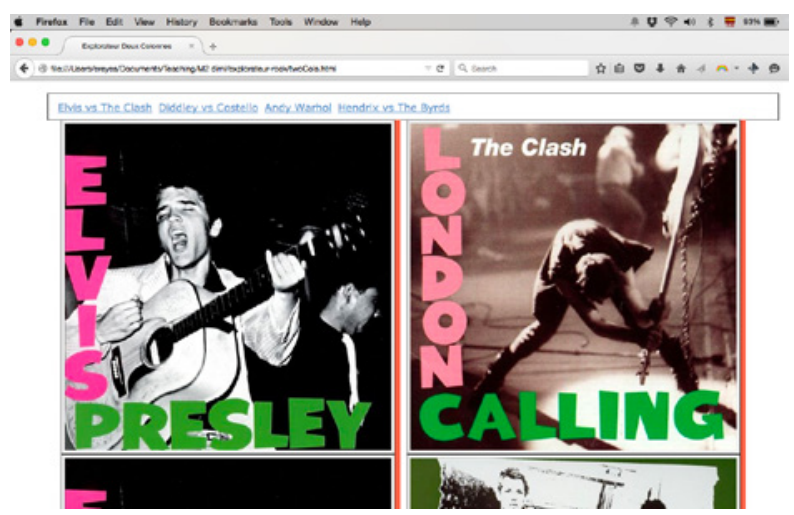

Source: Élaboration propre.

Dans le premier exemple, les valeurs chromatiques des images sont représentées comme des blocs de couleurs interactifs. Au survol de la souris sur chaque rectangle, l'image référencée s'affiche sur la partie droite de l'espace web. Ensuite, pour chaque image de pochette il est possible de visualiser une version sans couleurs, représentant uniquement les formes des figures dans l'image. Finalement, un lecteur de musique propose d'écouter le morceau sélectionné ${ }^{36}$.

Dans le deuxième exemple (l'image à droite), l'espace conçu est divisé en deux colonnes. Sur ces deux colonnes s'affichent les mêmes pochettes des disques et l'idée est que le chercheur parcoure, identifie et annote des points en commun entre deux images. À titre indicatif, l'interface contient, en haut de la page, des liens prédéfinis vers des similitudes et parodies classiques dans l'histoire du rock. Comme le cas précédent, l'intention était de motiver les étudiants avec des idées de fonctionnalités susceptibles d'évoluer. L'organisation sons ont un URL unique. Il est donc possible de les intégrer sur un site web. «https:// www.spotify.com>. 
en forme de colonnes rappelle qu'un espace web peut se diviser en plusieurs sous-espaces : multiples colonnes, multiples sous-fenêtres, multiples couches, et un espace vertical et horizontal presque infini (que l'on peut naviguer par les «ascenseurs» vers le bas ou vers la droite de la page).

Pour sa part, la réinterprétation des étudiants a était présentée sous forme de cinq mini-projets. Il est hors sujet d'exposer ici la méthodologie pédagogique, ni les attentes et déroulement du cours, mais nous souhaitons seulement discuter un exemple parmi ces cinq projets qui nous semble le plus adapté aux objectifs fixés au départ.

Le mini-projet Playlist ${ }^{37}$ propose un espace web interactif pour explorer la typographie de pochettes de disques. Le travail effectué en amont par les étudiants a consisté à prédécouper les lettres de logotypes de groupes et de titres d'albums pour les sauvegarder sous forme d'image. Ensuite, le visiteur peut composer un mot avec ces lettres. Le résultat est un mélange de styles typographiques mais qui permet de créer une «playlist» de chansons. Chaque lettre est associée à une chanson de l'album auquel elle fait référence. Finalement, le visiteur est invité à personnaliser la pochette de sa playlist selon trois critères: 1) un mosaïque d'images de pochettes de disques provenant des lettres choisies, 2) une couleur unie sélectionnée parmi les tonalités les plus représentatives des disques, 3) un espace de création pour déplacer librement les pochettes et les éléments graphiques.

37 Conçu et développé par Sophie Chapotat, Idrissa Diedhieu, Mickaël Duvernay, Tony Tran et Lucas Turuani. Accessible en ligne sur: «http://playlist.rocknshare.fr〉. 
Copie d'écran 5 - Images-interfaces du mini-projet Playlist

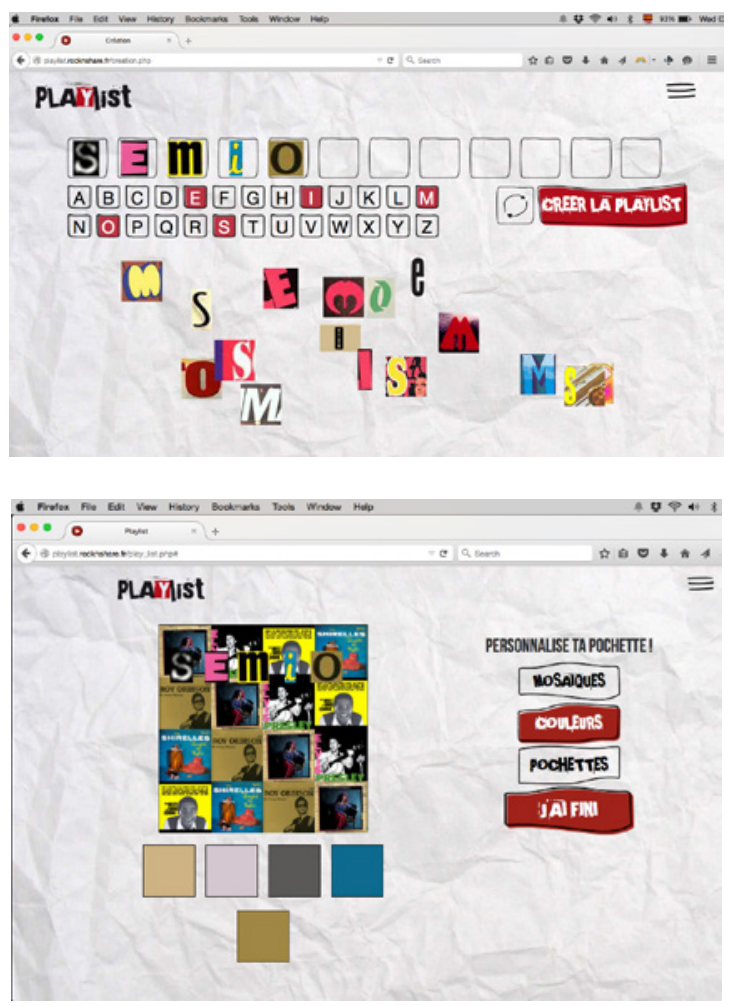

Source: élaboration des étudiants cités en bas de page

Malgré le fait que nos maquettes fonctionnelles d'imagesinterfaces, ainsi que celles conçues par des étudiants, restent des prototypes, nous pouvons les voir aussi comme une forme de apprentissage de notions de sémiotique visuelle et du design d'espaces web. Le point de vue que nous défendons est que les espaces web ne doivent pas être perçus forcément comme des environnements ayant un objectif donné ou pouvant prédéfinir les actions des visiteurs. Les espaces web peuvent constituer un espace d'exploration et de découverte; d'engagement et d'indétermination; de médiations entre un sujet d'étude et la 
manière de l'approcher. Ces espaces ne sont pas non plus des versions fixes; ils peuvent se revoir et faire évoluer.

En terme de compétences et méthodologie d'étude, nous pensons nécessaire le fait de développer des espaces originaux pour expérimenter des corpus de données. Les analyses classiques et les rapports documentaires gardent, certes, toute leur valeur et intérêt, mais dans une perspective du design et d'humanités numériques, la production et la création permettent de raisonner et réfléchir sur nos propres pratiques.

Dans nos travaux à futur nous envisageons de continuer nos expériences au niveau pédagogique et académique, en favorisant le travail collaboratif et multidisciplinaire, en partageant les données, les codes informatiques et les apprentissages acquis. La thématique de la musique rock est bien un exemple d'accès aux problématiques du design d'espaces web, mais d'autres corpus doivent se constituer, se raffiner et se partager.

\section{Conclusions: vers une sémiotique numérique}

Dans cette contribution nous avons présenté des expériences liées à la pratique de la recherche en sémiotique visuelle. Cette pratique a été approchée du point de vue de la production de ce qu'on appel des «espaces web». Pour nous, une page ou un site web est un espace web dans le sens où il s'insère dans un ensemble plus grand de ressources existantes et pouvant se visualiser grâce aux technologies web, notamment un navigateur, une adresse URL, un protocole de connexion et transfert, et des langages de format adaptés aux réseaux internet. 
La manière dont on peut penser et concevoir un espace web peut s'inspirer de la recherche en sémiotique visuelle. Nous pensons non seulement aux objets d'étude concrets (numérisations de peintures, de photographies, de BD, etc.) mais aussi aux méthodes d'analyse (vectorisation de formes, identification d'éléments plastiques, interprétation symbolique et figurative). De ce fait, un espace web peut acquérir la forme d'un environnement d'exploration qui intègre un discours complexe: différents types de ressources, leur quantification et métadonnées, les stratégies de digrammatisation et visualisation pour leur mise en interface.

Aux analyses traditionnelles en papier et documentaires, nous pensons que les espaces web peuvent valoriser la recherche en sémiotique. En même temps, les nouvelles images-interfaces qui surgissent nécessitent d'un cadre théorique pour leur réception et lecture. Une image-interface est la manière dont le discours complexe est représenté et actualisé. C'est un espace d'énonciation car le producteur choisi parmi des éléments existants et considère les contraintes techniques du web et les cadres de lecture des «utilisateurs». Ces utilisateurs sont mieux identifiés comme des «visiteurs» ou comme des «chercheurs»: des personnes qui naviguent et explorent sans avoir un «besoin» prédéfini.

Il nous semble que la sémiotique a un grand intérêt pour se interroger sur les possibilités de distribution et de documentation scientifique en ligne. Le web est devenu un espace puissant en termes techniques et presque ubiquitaire en terme d'accès grâce aux dispositifs mobiles. À la tendance de stabilisation de modèles de visualisation de données à l'ère du «big data», la sémiotique peut apporter une analyse des nouvelles formes de représentation mais aussi proposer des espaces inspirés de ses propres méthodologies; des espaces où 
l'utilisateur est un actant, un lecteur, un auteur, un énonciateur, un énonciataire, un producteur, un visiteur ou un public.

\section{RÉFÉRENCES}

BERNERS-LEE, T. Weaving the web. New York: Harper Collins, 1999.

BOLTER, J.; GRUSIN, R. Remediation. Cambridge, USA: MIT Press, 2000.

BUSH, V. As we may think. The Atlantic, Juillet, 1945.

CUBITT, S. The practice of light. Cambridge, USA: MIT Press, 2014.

DONDERO, M.G.; FONTANILLE, J. Des images a problèmes. Limoges : PUL, 2012.

DONDERO, M.G.; REYES, E. La substance du plan de l'expression de l'image. In : CONGRES DE L'ASSOCIATION FRANÇAISE DE SEMIOTIQUE. Juillet, 2015.

DRUCKER, J. Graphesis. Oxford: Oxford University Press, 2014.

FONTANILLE, J. Sémiotique du visible. Paris : PUF, 1995.

FONTANILLE, J. Pratiques sémiotiques. Limoges : PUL, 2008.

GREIMAS, A. Sémiotique figurative et sémiotique plastique. Actes Sémiotiques, v. 4, n. 60, 1984. 
GROUPE MU. Traité du signe visuel. Paris : Seuil, 1992.

GOOGLE OFFICIAL BLOG. We knew the web was big... 2008. Disponible: <http://googleblog.blogspot.com/2008/07/weknew-web-was-big.html>. Consulté: 13 déc. 2015.

LIMA, M. Visual complexity. New York: Princeton Architectural Press, 2011.

MANOVICH, L. 2010. What is visualization? The Journal of the Initiative for Digital Humanities, Media, and Culture, v. 2, n. 1, 2010.

MANOVICH, L. Media visualization: visual techniques for exploring large media collections. Media Studies Futures, 2011.

MANOVICH, L.; DOUGLASS, J. Visualizing temporal patterns in visual media. 2009. Disponible: 〈http://manovich.net/ index.php/projects/article-2009〉. Consulté: 13 déc. 2015.

McLUHAN, M. Understanding media. New York: McGraw Hill, 1964.

NELSON, T. A File Structure for the Complex. In: ACM 20 National Conference, 1965.

REYES, E. Ver lo visible: uso de técnicas digitales para el análisis visual. In: Virtualis, n. 5, 2012a.

REYES, E. Powell-Peralta: un análisis de tablas skateboard. In: 6eme. Colloque de l'AMESVE (Association Mexicaine de Sémiotique Visuelle et de l’Espace), Mexico, 2012b. 
REYES, E. La visualité de Nirvana. In : SEMINAIRE HISTORIE SOCIALE DU ROCK. Centre Malher, Paris, mai, 2013.

REYES, E. Explorations in Media Visualization. In: Proceedings of the ACM conference Hypertext'14. New York : ACM Press, 2014.

REYES, E. La datavisualisation comme image-interface. In : I2D : Information, données et documents. n. 2, 2015.

SONESSON, G. Methods and models in pictorial semiotics. Lund: Lund University Press, 1988.

THÜRLEMANN, F. Blumen-Mythos (1918) de P. Klee. In: HÉNAULT, A.; BEYAERT-GESLIN, A. Ateliers de sémiotique visuelle., Paris : PUF, 2004.

W3C. HTML5. 2014. Disponible : <http://www.w3.org/TR/ html5/>. Consulté: 13 déc. 2015.

Artigo recebido em setembro de 2015 e aprovado em dezembro de 2015.

Disponível em: http://seer.fclar.unesp.br/casa 